\title{
Prognostic Assessment in High-Grade Soft-Tissue Sarcoma Patients: A Comparison of Semantic Image Analysis and Radiomics
}

\author{
Jan C. Peeken ${ }^{1,2,3,4, *(D)}$, Jan Neumann ${ }^{5}\left(\mathbb{D}\right.$, Rebecca Asadpour ${ }^{1}$, Yannik Leonhardt ${ }^{5}$, Joao R. Moreira ${ }^{5}$, \\ Daniel S. Hippe ${ }^{6}$, Olena Klymenko ${ }^{1}$, Sarah C. Foreman ${ }^{5}$, Claudio E. von Schacky ${ }^{5}$, Matthew B. Spraker ${ }^{7}$, \\ Stephanie K. Schaub ${ }^{6}$, Hendrik Dapper ${ }^{1}$, Carolin Knebel ${ }^{8}$, Nina A. Mayr ${ }^{6}$, Henry C. Woodruff ${ }^{4,9}$ (D, \\ Philippe Lambin ${ }^{4,9}$, Matthew J. Nyflot ${ }^{6,10}$, Alexandra S. Gersing ${ }^{5}$ and Stephanie E. Combs ${ }^{1,2,3}$
}

check for

updates

Citation: Peeken, J.C.; Neumann, J.; Asadpour, R.; Leonhardt, Y.; Moreira, J.R.; Hippe, D.S.; Klymenko, O.; Foreman, S.C.; von Schacky, C.E.; Spraker, M.B.; et al. Prognostic Assessment in High-Grade Soft-Tissue Sarcoma Patients: A Comparison of Semantic Image Analysis and Radiomics. Cancers 2021, 13, 1929. https://doi.org/10.3390/ cancers13081929

Academic Editor: Dirk Clevert

Received: 1 March 2021

Accepted: 13 April 2021

Published: 16 April 2021

Publisher's Note: MDPI stays neutral with regard to jurisdictional claims in published maps and institutional affiliations.

Copyright: (c) 2021 by the authors. Licensee MDPI, Basel, Switzerland. This article is an open access article distributed under the terms and conditions of the Creative Commons Attribution (CC BY) license (https:// creativecommons.org/licenses/by/ $4.0 /)$.
1 Department of Radiation Oncology, Klinikum rechts der Isar, Technical University of Munich (TUM), Ismaninger Straße 22, 81675 Munich, Germany; rebecca.asadpour@tum.de (R.A.);

olena.klymenko@mri.tum.de (O.K.); hendrik.dapper@mri.tum.de (H.D.); stephanie.combs@tum.de (S.E.C.)

2 Institute of Radiation Medicine (IRM), Department of Radiation Sciences (DRS),

Helmholtz Zentrum München, 85764 München, Germany

3 Deutsches Konsortium für Translationale Krebsforschung (DKTK), Partner Site Munich, Germany

4 Department of Precision Medicine, GROW-School for Oncology and Developmental Biology, Maastricht University, 6200 MD Maastricht, The Netherlands; h.woodruff@maastrichtuniversity.nl (H.C.W.); philippe.lambin@maastrichtuniversity.nl (P.L.)

5 Department of Radiology, Klinikum rechts der Isar, Technical University of Munich (TUM), 81675 Munich, Germany; jan.neumann@tum.de (J.N.); yannik.leonhardt@tum.de (Y.L.); ricardounifesp73@gmail.com (J.R.M.); sarah.foreman@tum.de (S.C.F.); c.schacky@tum.de (C.E.v.S.); alexandra.gersing@tum.de (A.S.G.)

6 Department of Radiation Oncology, University of Washington, Seattle, WA 98195, USA; dhippe@uw.edu (D.S.H.); skschaub@uw.edu (S.K.S.); ninamayr@uw.edu (N.A.M.); nyflot@uw.edu (M.J.N.)

7 Department of Radiation Oncology, Washington University in St. Louis, St. Louis, MO 63110, USA; mspraker@wustl.edu

8 Department of Orthopedics and Sports Orthopedics, Klinikum rechts der Isar, Technical University of Munich (TUM), 81675 Munich, Germany; carolin.knebel@mri.tum.de

9 Department of Radiology and Nuclear Imaging, GROW-School for Oncology and Developmental Biology, Maastricht University Medical Centre, 6229 HX Maastricht, The Netherlands

10 Department of Radiology, University of Washington, Seattle, WA 98195, USA

* Correspondence: jan.peeken@tum.de; Tel.: +49-8941404501

Simple Summary: Soft-tissue sarcomas constitute a rare cancer type, with approximately $40 \%$ of patients experiencing disease recurrence. There is a need for a better identification of patients with especially aggressive tumors. Previous research demonstrated that the qualitative assessment of imaging data by radiologists ("semantic features") and the algorithm-based analysis of imaging data (termed "radiomics") may help to achieve a more thorough identification of patients at high risk for cancer-specific mortality. In this work, we compared the performance of predictions of patients' survival based on semantic features extracted by radiologists with a "radiomic" approach. While some semantic features were helpful to identify high-risk patients, the radiomic approach achieved an overall improved ability to identify patients at high risk. For the radiomic prediction, only one MRI sequence was sufficient and an MRI sequence without the need for contrast agent achieved good predictive performance.

Abstract: Background: In patients with soft-tissue sarcomas of the extremities, the treatment decision is currently regularly based on tumor grading and size. The imaging-based analysis may pose an alternative way to stratify patients' risk. In this work, we compared the value of MRI-based radiomics with expert-derived semantic imaging features for the prediction of overall survival (OS). Methods: Fat-saturated T2-weighted sequences (T2FS) and contrast-enhanced T1-weighted fatsaturated (T1FSGd) sequences were collected from two independent retrospective cohorts (training: 108 patients; testing: 71 patients). After preprocessing, 105 radiomic features were extracted. Semantic imaging features were determined by three independent radiologists. Three machine learning 
techniques (elastic net regression (ENR), least absolute shrinkage and selection operator, and random survival forest) were compared to predict OS. Results: ENR models achieved the best predictive performance. Histologies and clinical staging differed significantly between both cohorts. The semantic prognostic model achieved a predictive performance with a C-index of 0.58 within the test set. This was worse compared to a clinical staging system (C-index: 0.61 ) and the radiomic models (C-indices: T1FSGd: 0.64, T2FS: 0.63). Both radiomic models achieved significant patient stratification. Conclusions: T2FS and T1FSGd-based radiomic models outperformed semantic imaging features for prognostic assessment.

Keywords: radiomics; machine learning; soft-tissue sarcomas; radiology; MRI; tail sign; prognosis; elastic net regression

\section{Introduction}

Soft-tissue sarcomas (STS) constitute a rare malignant entity comprising $1 \%$ of all cancers [1]. Patient outcome and therapeutic management differ significantly between anatomic sites of the primary tumor (1). In patients with high-risk STS of the extremities, resection is commonly combined with neoadjuvant or adjuvant radiotherapy (RT) for improved local progression-free survival (LPFS) and overall survival (OS) [2,3]. In contrast to the high LPFS rates of up to $94 \%$, current therapy regimens achieve comparably low OS, with low distant progression-free survival (DPFS) rates [4-7].

There are large research efforts underway to find biomarkers for the prediction of therapy response, disease progression, and survival. Semantic imaging features have been shown to correlate with prognosis in multiple cancer entities [8-10]. In STS patients with diverse histologies, Crombé et al. demonstrated significant correlations of three semantic features (peritumoral enhancement, necrosis, heterogenous Tw2 signal intensity) with high tumor grading, OS, and DPFS [11].

The relatively novel field of imaging-based "radiomics" constitutes an alternative approach to characterize tissues, with the advantage of analyzing the whole tumor volume instead of only a focal biopsy sample. It is defined as an algorithm-based large-scale quantitative analysis of imaging features [12-15]. Such imaging biomarkers were shown to predict survival, tumor progression, spatial infiltration, and molecular aberrations in a multitude of cancer types [16-21]. In a recent publication, Spraker et al. did demonstrate a prognostic potential of contrast-enhanced and fat-saturated T1-weighted (T1FSGd) sequence-based radiomics in STS patients [22]. An earlier pilot study showed a predictive capability for distant metastases by applying radiomics to T1-weighted and T2-weighted fat-saturated (T2FS) sequences that were fused with ${ }^{18} \mathrm{~F}$-fluorodeoxyglucose positron emission tomography data [23].

The scope of this study was to compare the benefit of expert-derived semantic imaging features with radiomic models based on multiparametric MRI-scans, combining T1FSGd and T2FS sequences. The predictive value for OS was assessed and compared to clinical baseline models. The resulting models were validated in an external patient cohort. Finally, the importance of single semantic features was assessed.

\section{Materials and Methods}

\subsection{Patients}

Two independent patient cohorts from the University of Washington, Seattle, WA, USA (UW) and the Technical University of Munich, Munich, Germany (TUM) were used for radiomic model training and testing, respectively. Patient records of patients with STS of the extremities or trunk were analyzed for patients' age, grading, and TNM-staging. All patients received either preoperative, postoperative, or definitive RT, curative in intent, with or without chemotherapy. Exclusion criteria were low-grade, incomplete imaging data; previous RT; primary bone sarcomas; Ewing sarcomas; rhabdomyosarcomas; distant 
metastases at diagnosis (M1); and endoprosthesis-dependent MRI artifacts. See Figure S1 for a patient workflow. If an exclusion-relevant criterium was missing, the patient was excluded. In the final patient cohort, no modeling-specific data were missing. OS was calculated from the initial pathologic diagnosis to the time point of death or the time point of censoring. Data reporting follows the TRIPOD recommendations (Table S10: TRIPOD checklist) [24].

\subsection{Image Acquisition and Definition of Volume of Interests}

Each included patient received pre-RT MRI scans. See Table S2 for acquisition parameters and scan planes. Tumor segmentation was performed using MIM software version 6.6 (MIM Software Inc, Cleveland, USA), Eclipse 13.0 (Varian Medical Systems, Palo Alto, USA), iplan RT 4.1.2 (Brainlab, Munich, Germany), and 3D Slicer (3D Slicer, Version 4.8 stable release). All segmentations were transformed to masks. The primary tumor as the volume of interest (VOI) was manually segmented by JCP, by adapting existing expert segmentations from RT treatment planning in the TUM cohort. In the UW cohort, segmentation was performed by MBS, MM, JCP, and TC. Edematous changes were not included in the VOI. To compensate for operator-dependent bias, multiple delineations were performed for 21 randomly selected patients by three radiation oncologists (RA, MBS, JCP) in the UW cohort (see Figure 1). The DiceComputation module of 3D Slicer was used to calculate the Dice coefficient (DC) [25].

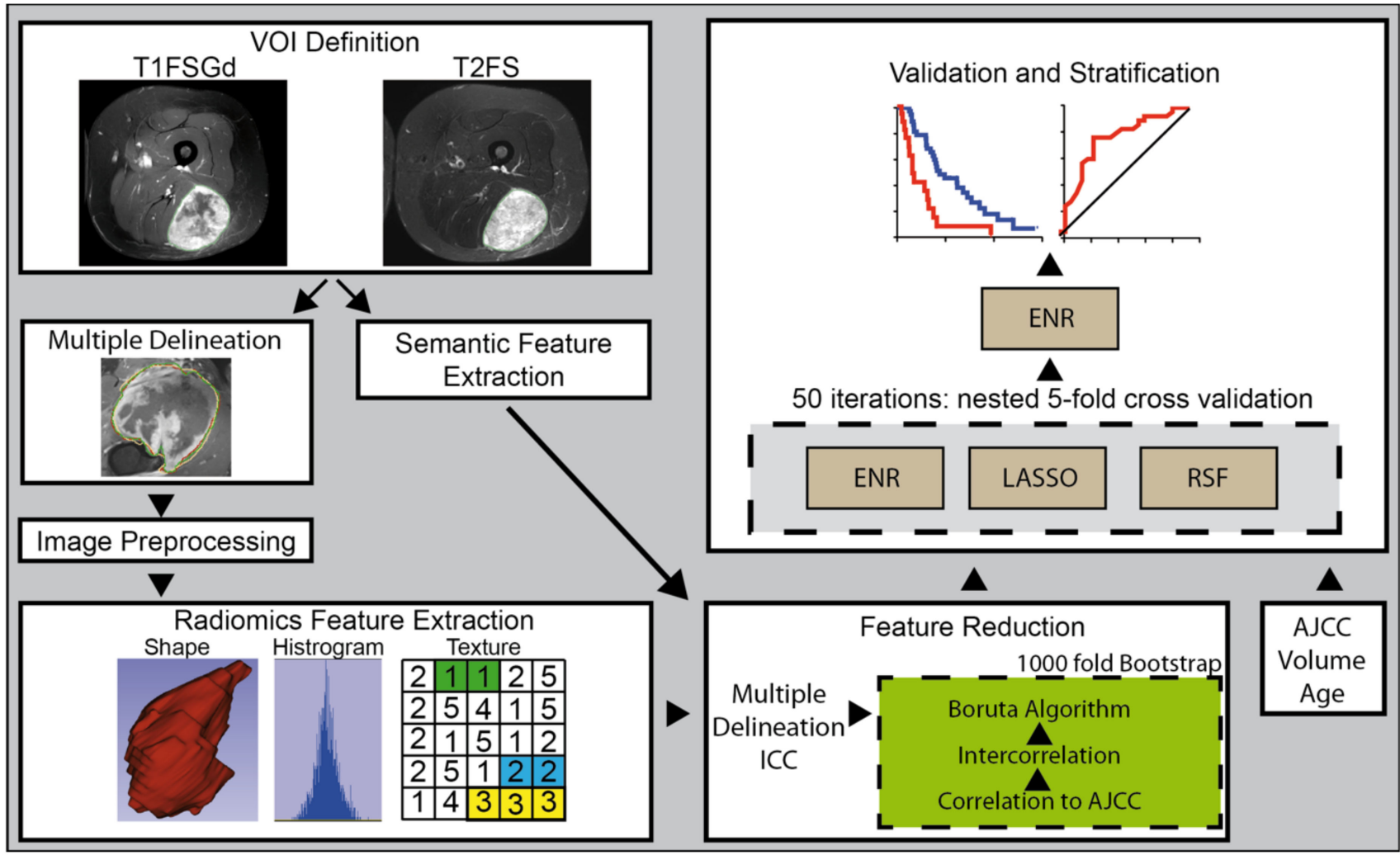

Figure 1. Radiomics Workflow. Abbreviations: AJCC: American Joint Committee on Cancer and the International Union for Cancer Control (8th edition), DCA: decision curve analysis, ENR: elastic net regression, ICC: intraclass coefficient, LASSO: least absolute shrinkage and selection operator, T1FSGd: T1-weighted fat-saturated with gadolinium, T2FS: T2-weighted fat-saturated, VOI: volume of interest.

\subsection{Image Preprocessing and Radiomic Feature Extraction}

N4ITK MRI bias field correction was applied to each imaging study using the Slicer3D implementation to compensate for non-uniform intensity caused by field inhomogeneity [26]. The pyradiomics package (Version 2.2) implemented in Python (3.7) was used for 
all preprocessing steps and radiomic feature extractions [27]. All radiomic features were calculated consistent with the Imaging Biomarker Standardization Initiative (IBSI) [28]. Preprocessing was conducted before image analysis. Due to the relative nature of MRI intensity values, image discretization was performed with a fixed bin width of 10 . Intensity normalization was performed by redistributing the image at the mean with the standard deviation and a scale of 100 . Isotropic resampling to a voxel size of $1 \times 1 \times 1 \mathrm{~mm}$ was performed by using Bspline interpolation. No voxel array shift was performed to be consistent with the IBSI guidelines. As current data point towards impaired reproducibility of filter-based features, we extracted features only from the original version of the image [29]. In sum, 105 features were extracted from the original image of each sequence within the segmented label map, including first-order features, shape features, and texture features. Texture features included "Gray Level Co-occurrence Matrix" (GLCM) features, "gray level size zone matrix" (GLSZM) features, "gray level run length matrix" (GLRLM) features, "neighboring gray tone difference matrix" (NGTDM) features, and "gray level dependence matrix" (GLDM) features, leading to a total feature number of 210. A detailed listing of extracted features is shown in Table S3.

\subsection{ComBat Batch Harmonization}

ComBatHarmonization has been proposed as a method for the correction of batch effects among multicenter radiomic cohorts [30,31]. Its value to improve reproducibility between different centers has been shown in multiple studies [32-34]. The additive and multiplicative batch effects on a given feature distribution are estimated using a maximum likelihood approach. We applied nonparametric ComBatHarmonization (https: / github. com/Jfortin1/ComBatHarmonization, accessed on 16 April 2020), correcting for MRI scanner models with mean site effects adjustment. We compensated for the MRI scanner type due to the small patient number.

\subsection{Semantic Imaging Features}

The MR imaging examinations were independently assessed by three radiologists ( 8 years, 7 years, and 3 years of experience in musculoskeletal radiology, respectively). The radiologists were blinded to the clinical information as well as the histological diagnosis. Two of the radiologists exclusively read imaging studies of one of the cohorts. The third radiologist, however, read image studies from both cohorts. Ten patients within the TUM cohorts were read by all three radiologists to assess the interrater agreement. The following radiological features were assessed in the study (see Table 1 for a description of all features) [35-38]: anatomical region of tumor (chest/back, leg, foot, arm, hand, gluteal/pelvic region), localization (epifascial, subfascial, or epi- and subfascial; intramuscular; intermuscular or inter-/intramuscular), tumor morphology (multinodular (more than one separate mass in the same region), mass-like (round or oval mass) or with superficial expansion along membranes/surfaces)), and tumor margins (well-defined, locally infiltrating or diffusely infiltrating). Moreover, on T1-weighted images with fat saturation and contrast enhancement, volume of contrast-enhancing tumor tissue (extent of enhancement $<1 / 3,1 / 3-2 / 3$, or $>2 / 3$ of tumor volume), enhancement pattern (homogeneous/inhomogeneous), presence of vascularization (present/absent), necrosis (present/absent), perilesional contrast enhancement (present/absent), and the tail sign (defined as a well-defined, pointed curvilinear formation at least $10 \mathrm{~mm}$ in length on T1FSGd images) were assessed. The maximal tumor diameter without tail sign (in $\mathrm{mm}$ ) was measured on the T1FSGd images with contrast enhancement. On the T2FS images, presence of perilesional edema (present/absent), diameter of edema (in $\mathrm{mm}$ ), extent of edema (diffuse or circumscribed), dominant T2FS signal intensity (hypointense/isointense/hyperintense), and dominant T2FS signal pattern (homogeneous or inhomogeneous) were graded. Before modeling features were one-hot encoded to dummy variables. 
Table 1. Description of all semantic features extracted radiologist for the description of soft-tissue sarcomas.

\begin{tabular}{cc}
\hline Feature & Description \\
\hline Anatomical region & 1: chest/back, 2: neck, 3: leg, 4: gluteal/pelvis 5: arm, 6: hand, 7: foot \\
\hline Localization & $\begin{array}{c}\text { 1: epifascial, 2: subfascial, 3: epi- and subfascial, 4: intramuscular, } \\
\text { 5: intermuscular; 6 intra- and intermuscular }\end{array}$ \\
\hline Image pattern & 1: multinodular, 2: mass-like round/oval, 3: superficial spread \\
\hline Borders & 1: well defined /pushing type, 2: focal infiltrating, 3: diffuse infiltrating \\
\hline Dominant STIR Signal intensity & 1: hypointense, 2: isointense, 3: hyperintense \\
\hline STIR Homogeneity & 1: homogenous, 2: inhomogenous \\
\hline Homogeneity of Tumor contrast enhancement & 1:<1/3 of the tumor, 2: 1/3-2/3, 3: $>2 / 3$ \\
\hline Tail sign & 1: homogeneous, 2: inhomogeneous \\
\hline Vascularization & 1: present, 0: absent \\
\hline Necrosis & 1: present, 0: absent \\
\hline perilesional Edema & 1: present, 0: absent \\
\hline perilesional Contrast enhancement & in mm \\
\hline Max diameter (in mm without tail) & in mm \\
\hline Edema diameter (in mm) & \begin{tabular}{c} 
1: ponsent, 2: uncertain \\
\hline
\end{tabular}
\end{tabular}

\subsection{Modeling Strategy}

Three common machine learning techniques established for survival analysis were trained and compared to predict OS: random survival forest (RSF), least absolute shrinkage and selection operator (LASSO), and elastic net regression (ENR) [39-41]. As a first feature reduction step, all features susceptible to variations in the subset of patients that received three independent segmentations were excluded. As a threshold, an intraclass correlation coefficient (ICC) $(3,1)$ of 0.8 was used. The remaining features (T1FSGd: 103, T2FS: 72) were used as input for the modeling pipeline. All three models were developed within the same pipeline. The pipeline combined (1.) additional feature reduction and (2.) model training (see Figure 1). (1.) The following feature reduction procedure was performed using 1000 bootstrap samples. Features correlated to the clinical American Joint Committee on Cancer and the International Union for Cancer Control (AJCC) (8th edition) staging groups defined by a Spearman correlation coefficient of greater than 0.8 were excluded [42]. Secondly, highly intercorrelated features defined by a Spearman correlation coefficient of greater than 0.8 were excluded. For the identified highly correlated feature pairs, the feature with the highest mean correlation to all remaining features was excluded. Thirdly, the Boruta algorithm was applied to filter the most relevant features [43,44]. The features were ranked according to the frequency of their selection in the 1000 bootstrap runs. The final feature set was defined as the top-ranking features. The final feature number per model was defined as the median feature number selected over all bootstrap runs.

To compare the performance of the three machine learning models, 50 iterations of 5 -fold nested cross-validation was performed using the UW cohort (referred to as "training cohort"). All three models were developed using the mlr3 package [45]. Hyperparameters were optimized using random search and 25 evaluations. The RSF was developed with 1000 trees. Hyperparameter optimization was conducted for node size (search space 3-20) and the number of input variables randomly chosen at each node (mtry) (search space 2-10). For ENR, alpha (search space 0.05-1.0) and lambda were optimized. For LASSO, alpha was set to 1 and lambda was optimized. No correction for unbalanced data was applied. After comparison of the modeling strategies (see 3.2) a final set of ENR models was retrained on the training cohort using 5 -fold cross-validation and tested on the TUM cohort (referred to as "testing cohort"). 
In total, three different radiomic models were developed: Radiomics-T1 based on T1FSGd-derived radiomic features, Radiomics-T2 based on T2FS-derived radiomic features, and Radiomics-T1T2 combining both feature sets. A semantic model (Semantic) was trained using the semantic features as input. Finally, a model combing semantic and radiomic features (Radiomics-T1T2+Semantic) was developed. Combined models were trained and tested as multivariate cox regression models using AJCC-stage, age, and the predictors of the developed models as an input. The concordance index (C-index) was calculated to assess model performance. The $95 \%$ confidence interval was estimated using 1000 fold bootstrapping.

To assess the influence of independent patient cohorts on model performance, we recalculated the final models on a new training set, mixing randomly chosen patients from both institutions with equal size and event numbers compared to the original training cohort. The remaining mixed patients were used as a test cohort.

\subsection{Statistical Analysis}

Statistical analysis and modeling were performed using R (version 3.4.0, R core team, Vienna, Austria). See Table S4 for R packages and versions. Fleiss Kappa and ICC were calculated to test for interrater agreement. Kaplan-Meier survival curves were generated to analyze stratified patient subgroups. The cutoff to split patients into low-risk and high-risk patients was defined as the median of the predictors in the training set. Statistical significance was tested using the Log-rank test. Time-dependent area under the receiver operating characteristic (ROC) curve (AUC) and calibration curves were plotted to characterize model performances. Bonferroni correction was performed in cases of multiple testing as specified. A $p$-value below 0.05 was regarded as significant.

\section{Results}

\subsection{Patient Characteristics, Histology and VOI Definition}

Overall, patient demographics were similar between both cohorts (Table 2); however, STS histologies were different between the cohorts $(p<0.001)$ (Table S1). Pleomorphic sarcoma was the most frequent histology in both groups, although with a larger proportion in the training set (training: 45\%, testing: 34\%). The second and third most frequent histologies were leiomyosarcoma $(11 \%)$ and spindle cell carcinoma $(10 \%)$ in the training cohort and myxofibrosarcoma (18\%) and synovial sarcoma (13\%) in the testing cohort. There were more unfavorable characteristics in the testing cohort with a larger proportion of AJCC stage 3 patients and 5 patients $(7 \%)$ treated in a recurrent setting. Significantly more patients from the training cohort received chemotherapy. In the testing cohort, the delivered total RT dose was significantly higher than in the training cohort and a higher number of patients received definitive RT ( $6 \%$ vs 1\%). There was an overall high similarity between multiple tumor target volume delineations performed by the three independent operators with a mean Dice similarity coefficient (DSC) of 0.92 (range (min-max): 0.81-0.96).

\subsection{Interrater Agreement of Semantic Imaging Features}

Ten randomly chosen patients were read by the three independent radiologists. Nominal and ordinal features achieved a median Fleiss Kappa of 0.524 (range: 0.035-1.00). Overall, five features achieved a "substantial/good" agreement (Kappa >0.60), five features achieved a "moderate" agreement (0.40-0.60), and three features achieved only "slight/fair" agreements (0.00-0.40), as defined by Altmann and Landis [46,47]. The two continuous measures were correlated with a median ICC of 0.833 (range: 0.138-0.846). Table S5 displays all Kappa and ICC values for each feature. 
Table 2. Patient demographics, outcomes, and treatment specifics.

\begin{tabular}{|c|c|c|c|c|c|}
\hline Institution & & Testing Cohort & Training Cohort & $p$-Value ${ }^{1}$ & $p$-Value Adjusted ${ }^{1}$ \\
\hline Accrual time & & 2010-2016 & 2007-2015 & & \\
\hline \multirow[t]{3}{*}{ Total Patients } & & $71 \mathrm{p}$ & $108 \mathrm{p}$ & & \\
\hline & Primary & $66 \mathrm{p}(93 \%)$ & 108 p $(100 \%)$ & $<0.001^{*}$ & $<0.001 *$ \\
\hline & Recurrent & $5 \mathrm{p}(7 \%)$ & $0 \mathrm{p}$ & & \\
\hline \multirow[t]{3}{*}{ Location } & Lower Extremity & $56 \mathrm{p}(79 \%)$ & $75 \mathrm{p}(70 \%)$ & 0.36 & 1.0 \\
\hline & Upper Extremity & $10 \mathrm{p}(14 \%)$ & $17 \mathrm{p}(16 \%)$ & & \\
\hline & Trunk & $5 \mathrm{p}(7 \%)$ & $16 \mathrm{p}(14 \%)$ & & \\
\hline Age & & m 57 (r 17-87) & m 53.7 (r 19.1-88.5) & 0.16 & 1.0 \\
\hline \multirow[t]{3}{*}{ Gender } & female & $35 \mathrm{p}(49 \%)$ & $29(27 \%)$ & 0.005 * & 0.078 \\
\hline & male & $36 \mathrm{p}(51 \%)$ & $76(70 \%)$ & & \\
\hline & unknown & $0 \mathrm{p}$ & $3 \mathrm{p}(3 \%)$ & & \\
\hline \multirow[t]{4}{*}{ T-stage ${ }^{2}$} & 1 & $4 \mathrm{p}(6 \%)$ & $9 \mathrm{p}(8 \%)$ & 0.40 & 1.0 \\
\hline & 2 & $30 \mathrm{p}(42 \%)$ & $32 \mathrm{p}(30 \%)$ & & \\
\hline & 3 & $23 \mathrm{p}(32 \%)$ & $41 \mathrm{p}(38 \%)$ & & \\
\hline & 4 & $14 \mathrm{p}(20 \%)$ & $26 \mathrm{p}(24 \%)$ & & \\
\hline \multirow[t]{2}{*}{ M-stage $^{2}$} & 0 & $71 \mathrm{p}(100 \%)$ & $108 \mathrm{p}(100 \%)$ & - & - \\
\hline & 1 & $0 \mathrm{p}(0 \%)$ & $0 \mathrm{p}(0 \%)$ & & \\
\hline \multirow[t]{2}{*}{ N-stage ${ }^{2}$} & 0 & 69 p (97\%) & $108 \mathrm{p}(100 \%)$ & 0.16 & 1.0 \\
\hline & 1 & $2 \mathrm{p}(3 \%)$ & $0 \mathrm{p}$ & & \\
\hline \multirow[t]{3}{*}{ Grading $^{3}$} & 1 & $0 \mathrm{p}(0 \%)$ & $0 \mathrm{p}(0 \%)$ & 0.88 & 1.0 \\
\hline & 2 & $28 \mathrm{p}(39 \%)$ & $44 \mathrm{p}(40 \%)$ & & \\
\hline & 3 & $43 \mathrm{p}(51 \%)$ & $64 \mathrm{p}(60 \%)$ & & \\
\hline \multirow[t]{3}{*}{ AJCC-Stage $^{2}$} & IIA & $9 \mathrm{p}(13 \%)$ & $15(14 \%)$ & $0.0025 *$ & $0.045^{*}$ \\
\hline & IIB & $4 \mathrm{p}(6 \%)$ & $32(29 \%)$ & & \\
\hline & III & $48 \mathrm{p}(68 \%)$ & $61(58 \%)$ & & \\
\hline \multirow[t]{4}{*}{ Margin-status } & positive & $12 \mathrm{p}(17 \%)$ & $28 \mathrm{p}(26 \%)$ & 0.011 & 0.18 \\
\hline & negative & $53 \mathrm{p}(75 \%)$ & $76 \mathrm{p}(70 \%)$ & & \\
\hline & unknown & $2 \mathrm{p}(3 \%)$ & $3 p(3 \%)$ & & \\
\hline & no resection & $4 \mathrm{p}(6 \%)$ & $1 \mathrm{p}(1 \%)$ & & \\
\hline \multirow[t]{3}{*}{ RT type } & post-operative & $15 \mathrm{p}(21 \%)$ & $32(29 \%)$ & $<0.001 *$ & $0.007 *$ \\
\hline & neoadjuvant & $52 \mathrm{p}(72 \%)$ & $75 \mathrm{p}(70 \%)$ & & \\
\hline & definitive & $4 \mathrm{p}(6 \%)$ & $1 \mathrm{p}(1 \%)$ & & \\
\hline Total RT Dose & & $\begin{array}{c}\text { m } 50 \text { Gy(r 28-70 } \\
\text { Gy) }\end{array}$ & $\begin{array}{l}\text { m 50 Gy(r } \\
38-50 G y)\end{array}$ & $<0.001 *$ & $<0.001 *$ \\
\hline Chemotherapy & & $3 / 71 \mathrm{p}(4 \%)$ & $64 \mathrm{p} \mathrm{(59 \% )}$ & $<0.001 *$ & $<0.001 *$ \\
\hline Median OS & & 40.1 (r 6.0-105.5) & 39.9 (r 4.2-130.4) & 0.53 & 1.0 \\
\hline
\end{tabular}

Abbreviations: * : $p$-value < 0.05, AJCC: American Joint Committee on Cancer and the International Union for Cancer Control (8th edition), m: median, p: patients, r: range, RT: radiation therapy, ${ }^{1}$ Wilcoxon rank-sum test for continuous and ordinal variables, Fisher's exact test for nominal variables, log-rank test for comparison of survival times. Corrected for multiple testing by Bonferroni correction (" $p$-value adjusted"). ${ }^{2}$ Following AJCC staging system 8th edition [42]. ${ }^{3}$ According to the French Federation of Cancer Centers Sarcoma Group (FNCLCC). 


\subsection{Comparison of Semantic Imaging Features and Radiomics for Prediction of Overall Survival}

The feature reduction pipeline yielded a median number of 12 (Radiomics-T1, range: 4-23), 10 (Radiomics-T2, range: 3-16), 11 (Radiomics-T1T2, range: 7-27), and 9 (Semantic, range: 3-16) features that were used for the prediction models. Radiomics-T1T2+Semantic combined features of Radiomics-T1T2 and Semantic. The selected features are listed in Table S7.

Three ML modeling strategies were applied and compared to predict OS. The ML techniques were ranked in order of the predictive performance in the external crossvalidation folds for each model. ENR, RSF, and LASSO achieved a mean rank of 1.4, 2.2, and 2.4, respectively. Table S6 lists the C-indices per ML technique and feature set. Due to the overall better outcome of the ENR model, it was applied for further analyses and validated on the independent test set. See Figure 2 for the respective $C$-index values.

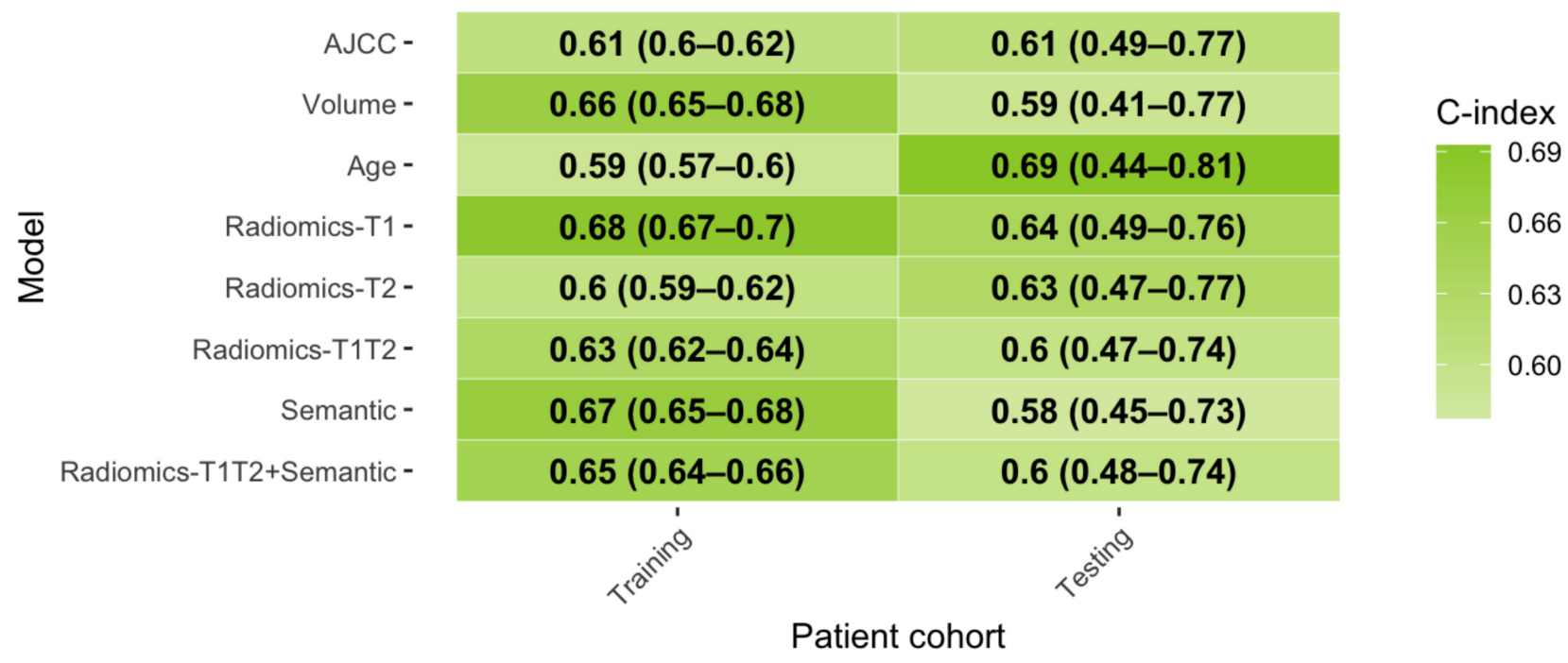

Figure 2. Prognostic performance of developed ENR models. Abbreviations: AJCC: American Joint Committee on Cancer and the International Union for Cancer Control (8th edition), C-index: concordance-index, OS: overall survival.

During nested cross-validation within the training set, Radiomics-T1 achieved a superior performance (C-index: 0.68) compared to Radiomics-T2 (C-index: 0.60). In comparison, the Semantic model achieved a performance comparable to Radiomics-T1 in the training set (C-index: 0.67).

In the external test set, however, both radiomic models performed similarly (RadiomicsT1: C-index: 0.64, Radiomics-T2: 0.63). Combining both feature sets (Radiomics-T1T2) did not trigger an improved testing performance (C-index: 0.60). The Semantic model failed to reproduce the predictive performance from the training set (C-index: 0.58). A model combining all imaging features Radiomics-T1T2+Semantic did not improve performance further (C-index:0.6). For comparison, three clinical baseline models were computed. The AJCC staging system (C-index: 0.61), and tumor volume (C-index: 0.59) showed worse performance compared to the radiomic models in the test set. Age achieved the highest C-index (0.69) among all predictors in the test set. Figures S2 and S3 depict the time-dependent AUC and calibration curves, respectively.

The propensity to achieve patient stratification was evaluated using Kaplan-Meier analysis (Figure 3). In the testing cohort, all three radiomic models achieved a significant separation of survival curves (curves were split at the median of the training cohort predictors). For Semantic, Radiomics-T1T2+Semantic, Volume, and Age, there was no significant risk stratification. For $A J C C$, a trend towards significance could be observed $(p=0.0532)$. 

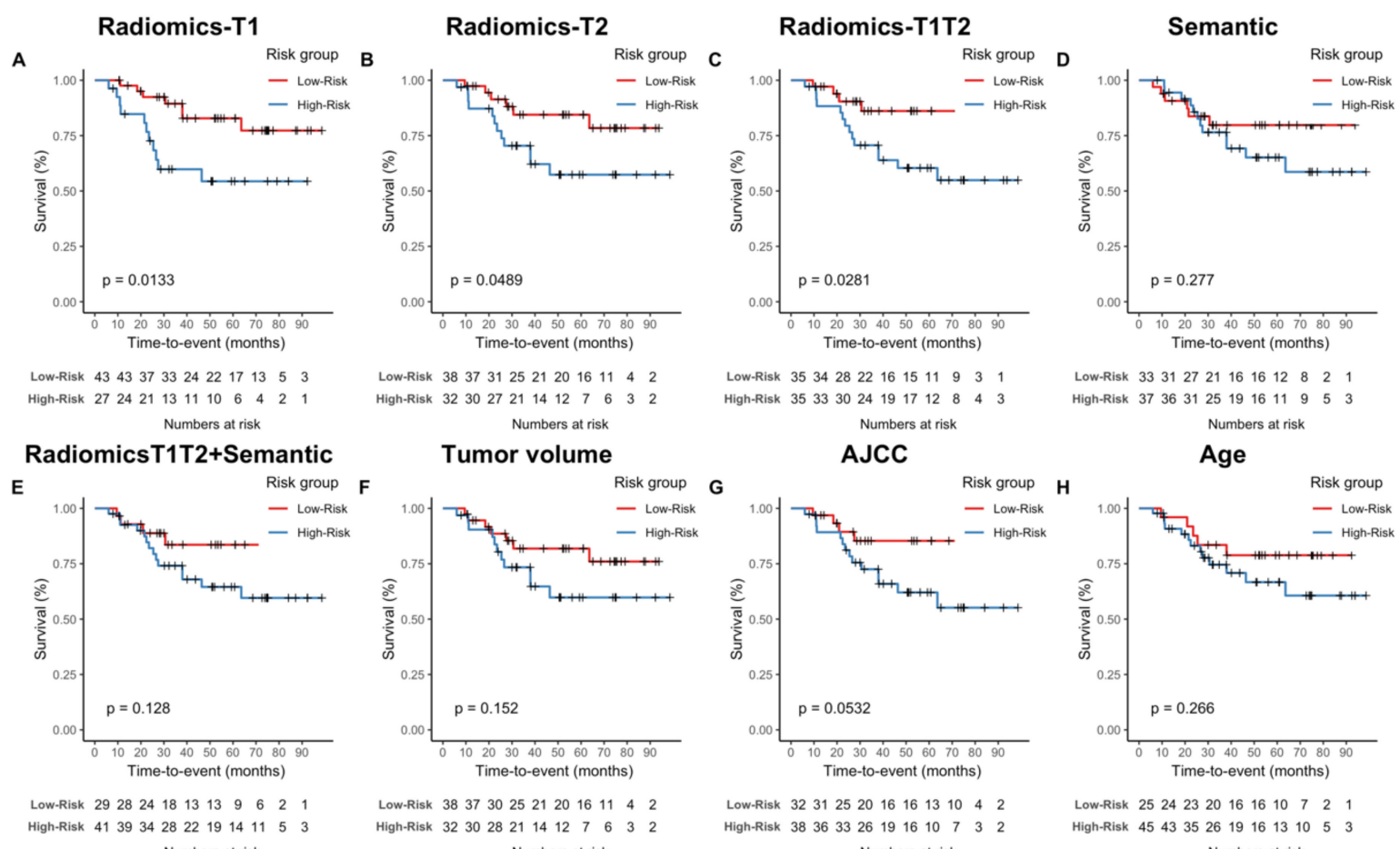

High-Risk $323027211412 \quad 7 \quad 6 \quad 3 \quad 2$

Numbers at risk

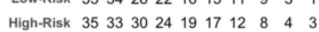

Low-Risk $33312721161612 \quad 8 \quad 2 \quad 1$

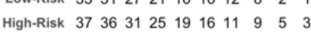

Tumor volume Numbers at risk Numbers at risk

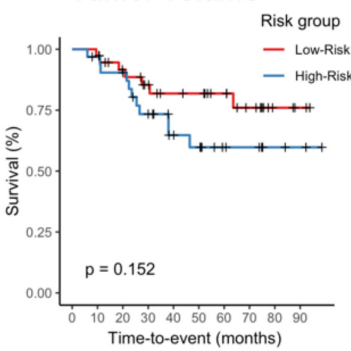

AJCC

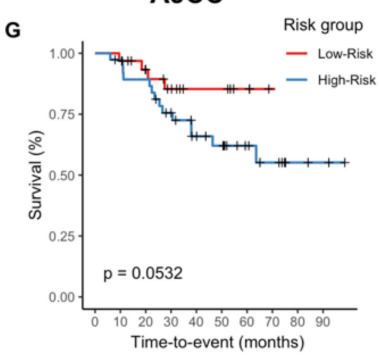

Age

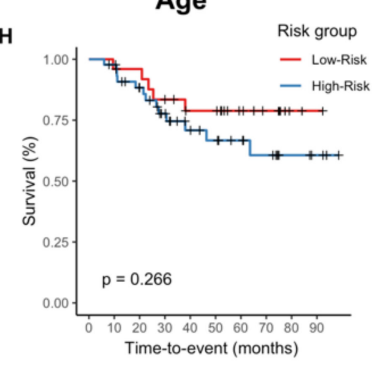

ow-Risk $3837302521201611 \quad 4$ High-Risk $32 \begin{array}{lllllllll}30 & 28 & 21 & 14 & 12 & 7 & 6 & 3 & 2\end{array}$

Numbers at risk

Numbers at risk

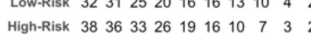

Numbers at risk
Low-Risk 252423201616107221 High-Risk $4543352619161310 \quad 5 \quad 3$

Figure 3. Kaplan-Meier survival analyses of developed models in the testing cohort. (A-F) Kaplan-Meier survival curves. Cohorts were split based on the median predictor value determined on the training cohort. $(\mathbf{G}, \mathbf{H})$ As a consequence, the AJCC staging system was split between stage III and stages IIA/B.

\subsection{Relevance of Combined Clinical-Imaging Models}

To test for a potential incremental benefit, the radiomic and semantic models were combined with the AJCC staging system and patients' age (Figure 4). AJCC + Age alone achieved the best performance in the test set so far (C-index: 0.71). Adding the Radiomics-T2 model improved the predictive performance further by +0.02 (Radiomics-T2+AJCC + Age: C-index: 0.73). Models combining AJCC and age with the Semantic model (C-index: 0.62) or the Radiomics-T1 model failed to increase performance (C-index: 0.67). The combined Radiomics-T2+AJCC + Age model achieved significant patient stratification in Kaplan-Meier analysis (Figure 5). The mean time-dependent AUC was 0.79.

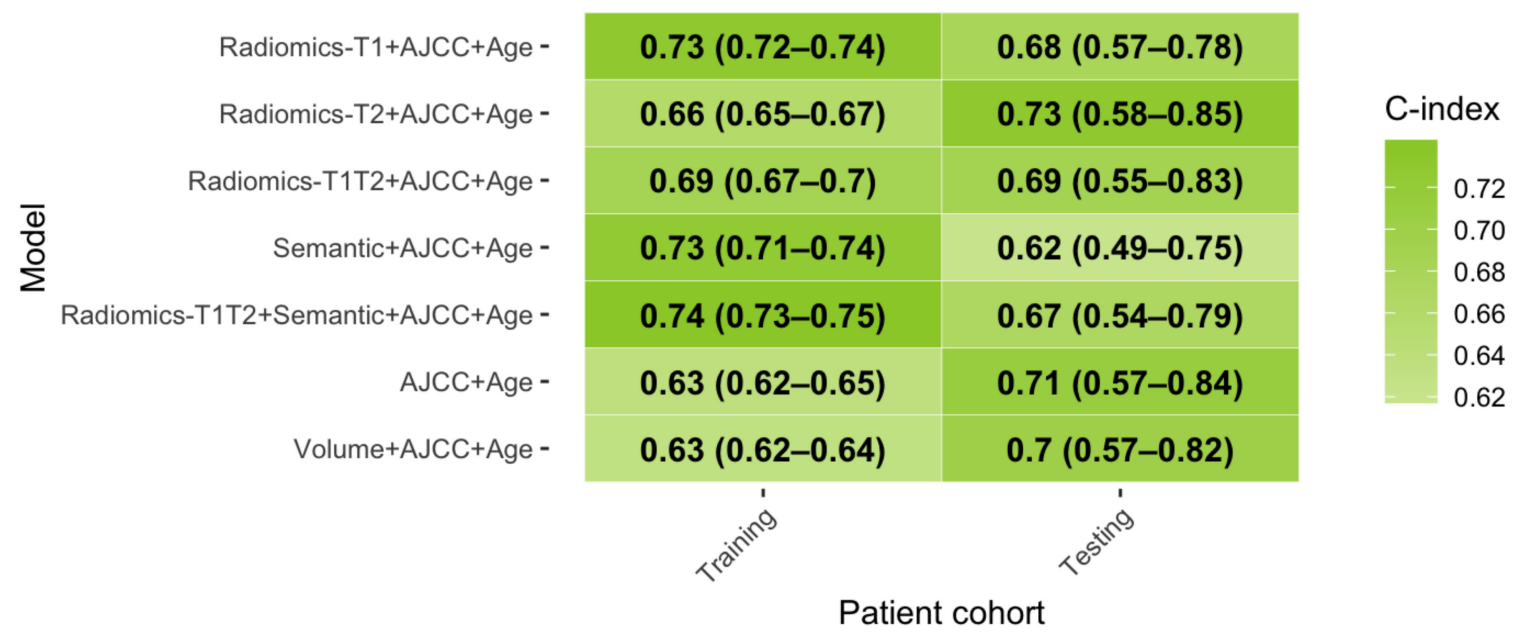

Figure 4. Prognostic performance of combined models. Abbreviations: AJCC: American Joint Committee on Cancer and the International Union for Cancer Control (8th edition), C-index: concordance-index, OS: overall survival. 

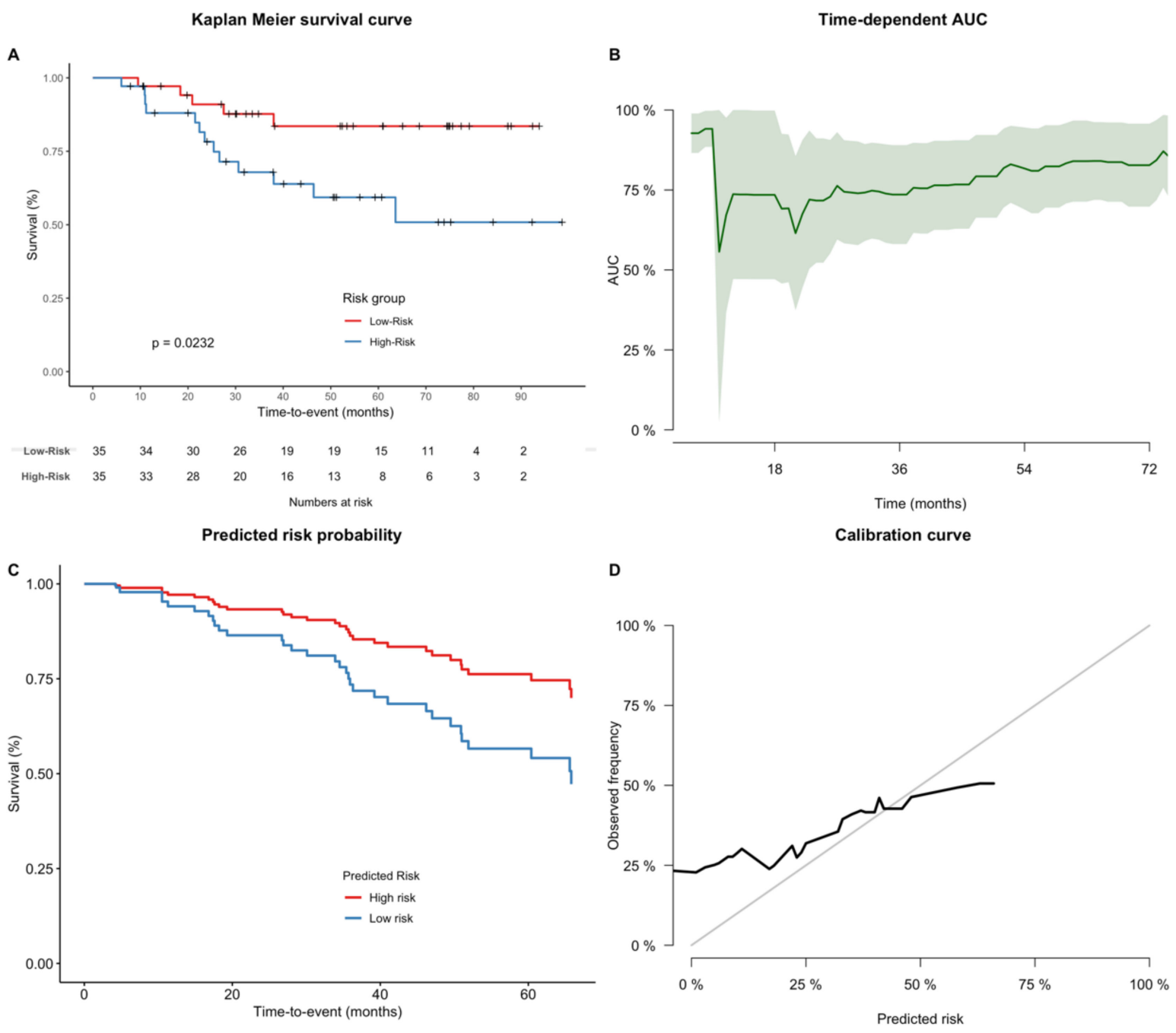

Figure 5. Prognostic performance of the Radiomics-T2+AJCC+Age model in the testing cohort. (A) Kaplan-Meier survival curve. Cohorts were split based on the median predictor value determined on the training cohort. (B) Time-dependent area under the receiver operating curve (AUC). (C) Predicted survival probabilities of the Cox proportional hazards model. (D) Calibration curve of the Cox proportional hazards model.

\subsection{Relevance of Single Imaging Parameters}

To investigate the prognostic value of isolated semantic features, univariate Cox proportional hazards regression was performed on the combined cohort (Table 3). Three features, including "maximal diameter without tail" $(p=0.022)$, "necrosis" $(p=0.039)$, and "edema perilesional" ( $p=0.043)$, were significantly associated with OS (without correction for multiple testing). When testing for an interaction between patient cohorts, none of the interactions reached statistical significance.

All semantic parameters that were found to be significant in the combined cohort were also included in the final feature reduction set. Besides, the parameters "epifascial and intramuscular location", "contrast enhancement perilesional", as well as the anatomic location "leg" were selected. See Table S7 for the coefficients of the final models. Figure 6 shows two exemplary cases.

In the Radiomics-T1 model, only the features "Firstorder-Mean" and "Shape-SurfaceArea" retained non-zero coefficients. The Radiomics-T2 model included several GLSZM and GLDM based features with non-zero coefficients. 
Table 3. Cox proportional hazards regression of semantic features for patients' overall survival.

\begin{tabular}{ccc}
\hline & \multicolumn{2}{c}{ Combined Cohort } \\
\hline Feature & HR (95\% CI) & $p$-Value \\
\hline Anatomic region & $0.58(0.33-1)$ & 0.067 \\
\hline Localization & $1.2(0.95-1.5)$ & 0.12 \\
\hline Image pattern & $0.94(0.59-1.5)$ & 0.8 \\
\hline Borders & $1.3(0.86-1.9)$ & 0.22 \\
\hline Maximal diameter without tail (in $\mathbf{m m})$ & $\mathbf{1 ~ ( 1 - 1 )}$ & $\mathbf{0 . 0 2 2}$ \\
\hline Dominant STIR signal intensity & $1.3(0.45-3.5)$ & 0.66 \\
\hline STIR homogeneity & $1.5(0.74-2.9)$ & 0.27 \\
\hline Tumor contrast enhancement & $0.74(0.52-1.1)$ & 0.1 \\
\hline Homogeneity of Tumor contrast & $1(0.54-1.9)$ & 0.98 \\
enhancement & $1.5(0.86-2.6)$ & 0.16 \\
\hline Tail sign & $0.95(0.47-1.9)$ & 0.88 \\
\hline Vascularization & $\mathbf{1 . 9}(\mathbf{1 - 3 . 6 )}$ & $\mathbf{0 . 0 3 9}$ \\
\hline Necrosis & $1.1(0.6-1.9)$ & 0.81 \\
\hline Contrast enhancement perilesional & $1.5(0.85-2.6)$ & $\mathbf{0 . 0 4 3}$ \\
\hline Edema perilesional (in mm) & 0.16 \\
\hline
\end{tabular}

Univariate Cox proportional hazards regression was performed for semantic imaging features. Significant factors are written in bold. Depicted $p$-values were not corrected for multiple testing. Abbreviations: 95\% CI: 95\% confidence interval.

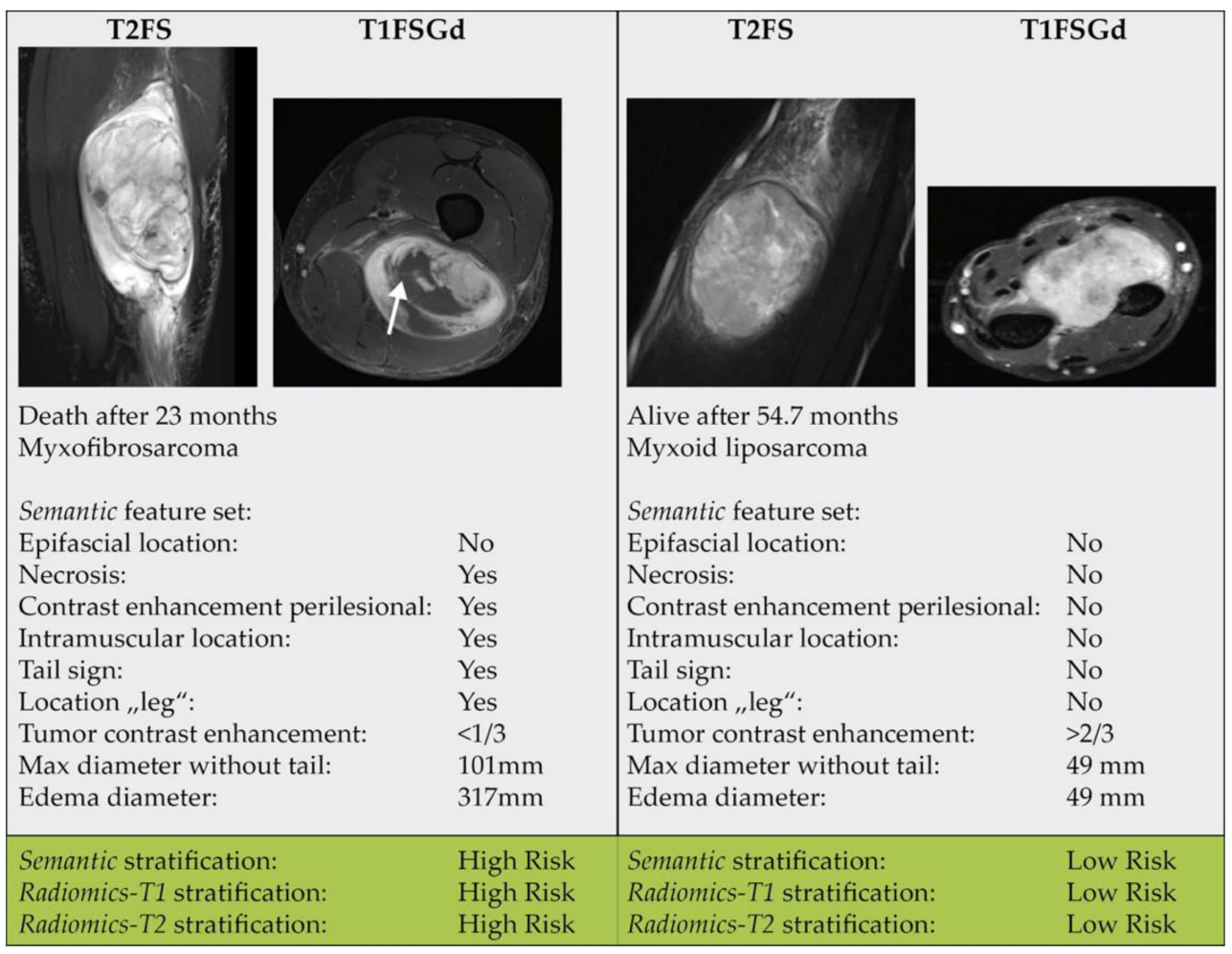

Figure 6. Two exemplary patient cases. 


\subsection{Analysis of Model Calibration}

Besides the C-index, we analyzed calibration curves of the developed models. Table S8 depicts Brier scores of all models. AJCC and Age had the lowest Brier scores (24 and 30, respectively). AJCC showed the most monotonous slope. Radiomic models showed Brier scores ranging from 88 to 114, while models comprising semantic features had the worst calibrations (Brier scores from 370-702). For combined models, however, the predicted risk showed a better correlation with observed frequency with lower Brier scores (Brier scores from 37 to 56) (Figure 5D, Figure S4). For all models, larger predicted risks were not well correlated to high observed frequencies.

\subsection{Assessment of the Impact of the Independence of the Test Cohort}

Retraining and testing of prediction models on randomly selected training and testing cohorts combining patients form both institutions led to a better reproducibility of the developed models. Both radiomic models Radiomics-T1 and Radiomics-T2 achieved a testing AUC of 0.63 , which was equal to the training performance. Interestingly, the Semantic model also achieved a higher reproducibility, with a testing AUC of 0.63 and a difference of -0.01 compared to the training set.

\section{Discussion}

In this work, we demonstrated that a standardized semantic prognostic model predicted survival with moderate performance. Radiomic models achieved an added benefit in predicting OS relative to semantic features. Interestingly, the performance of Radiomics$T 1$ and Radiomics-T2 was comparable in terms of C-index. Both models achieved significant risk stratifications in the testing cohort. Importantly, combining the T1FSGd and T2FS radiomic feature sets with or without the semantic features did not trigger an additional benefit. The best combined model using T2FS-based radiomics features (Radiomics-T2 $+A J C C+A g e$ ) achieved a modest incremental benefit above the clinical model $(A J C C+A g e)$ alone.

Multiple studies previously demonstrated significant associations of semantic imaging features with prognosis in STS patients. For instance, in a previous study, we demonstrated that semantic features, such as tumor size, septa thickness, contrast enhancement could distinguish atypical lipomatous tumors from benign lipomas [38]. The presence of perilesional edema and T2 heterogeneity of liposarcomas detected with MRI predicted pulmonary metastases in a previous study [48]. Moreover, perilesional edema detected on MR images of myxofibrosarcomas correlated significantly with a poor OS rate [49]. The "tail sign" describing tumor cell infiltrations extending from the primary tumor along the deep fascia has been shown to correlate with LPFS in myxofibrosarcoma and undifferentiated sarcomas [37]. In a non-histology-specific STS cohort, the three semantic features found by Crombé et al. (peritumoral enhancement, necrosis, heterogenous T2-weighted signal intensity) were also correlated to OS and DPFS [11]. In our analysis, necrosis was associated with worse survival as well. Besides necrosis, peritumoral enhancement and the tail sign were selected into the final Semantic feature set, signaling a predictive relevance in our study, too.

In the univariate analysis, we could identify significant prognostic semantic features. However, several factors may have contributed to negatively influencing the predictive performance of the developed combined semantic prediction model. First, a substantial proportion of the semantic features achieved only moderate interrater agreement and may have hindered effective reproduction in the testing cohort. Second, semantic feature assessment may have been impaired by image acquisition and reconstruction parameters as it is known for radiomic features. ComBatHarmonization has been proposed to reduce the variability of radiomic features [33]. Potential novel standardization techniques may help to harmonize between readers and/or imaging acquisition characteristics. Third, with the availability of only two distinct sequences and only one high resolution plane orientation per sequence, the radiologists' assessment was limited compared to a clinical 
setting. Fourth, semantic properties may differ significantly between different histologies of STS. As a consequence, the prognostic value of each feature may be different depending on the histological subtype, too. This may be of particular importance as both cohorts showed a different histological distribution of histologies. This could have impaired a better performance. As a consequence, building a Semantic "histology-agnostic" prediction model may simply not be feasible. Interestingly, Radiomic models seem to extract a proportion of histology-agnostic information. Regardless, histology-specific models may be superior for patient stratification and should be investigated in the future with the expansion of our multi-institutional database, given the rarity of STS overall and the further refinement with each particular histology with over 150 different subtypes. Alternatively, if a sufficiently large cohort would be available, histology could also be added as a predictive variable itself.

The significantly improved reproducibility of the Semantic model and, to a lesser extent, the Radiomic models in the non-independent training and testing cohort (Table S9) demonstrated the impact of differing treatment, acquisition, and patient characteristics between cohorts. This validation, however, only corresponds to a TRIPOD type II validation, as differences between the cohorts become mitigated following randomized splitting of the training and testing cohort $[24,50]$. The usage of independent cohorts as performed in the main results corresponds to a TRIPOD type III validation, yielding a better estimate for generalizability.

In our previous study, we demonstrated the feasibility of radiomic-based prognostic risk assessment based on planning CT data, despite its inferior soft-tissue resolution [51]. In a different study, we showed the general propensity to predict OS based on T1FSGd MRI sequences using radiomics. The final model achieved a C-index of 0.68 in the independent test set [22]. In this work, we could now demonstrate that by using T2FS sequences, a similar prognostic value can be achieved without the need for contrast agent administration. However, the reported performance of our MRI models and the incremental benefit above a clinical model was lower compared to the previous results. This may be reasoned by the more stringent selection of patients based on clinical criteria (e.g., exclusion of low-grade STS and non-extremity/trunk locations), and most importantly the simultaneous presence of T1FSGd and T2FS MRI sequences leading to a 34\% smaller training cohort (see the patient workflow in Figure S1).

Combing semantic and radiomic features did not improve the prediction of survival. This may be explained by the fact that radiomic features may at least partly encode tumorspecific semantic imaging features. Moreover, the total relevance of semantic features appeared to be inferior to the radiomic features when comparing model performances.

Model calibration among solely imaging-based models was suboptimal. By combing imaging models with clinical features, model calibration could be improved. As a consequence, future models should be combined with known clinical characteristics. This way, the best predictive performance and calibration can be achieved.

Improved pretherapeutic prognostic assessment of patients' risk for systemic progression or death may help to individualize treatment regimens. Current therapy regimens of high-grade STS achieve a good LPFS by combining resection and radiotherapy. DPFS and OS, however, remain comparably low [4]. Multiple studies have analyzed the use of additional systemic therapies. For instance, multiagent chemotherapy was recently shown to be significantly associated with improved OS in a large meta-analysis of 22 studies encompassing 5044 patients [52]. However, the toxicity of these chemotherapy regimens is substantial and the total outcome remains unfavorable. Novel systemic treatment agents could be an alternative and are currently under investigation in clinical trials [53,54]. Regarding the high mutational burden of some STS entities, immunotherapeutic checkpoint inhibition may be a further option for a systemic therapy modification, which is currently being tested in the phase-II Sarc032 trial using Pembrolizumab (NCT03092323) [55]. Other molecular targeted agents, such as the MDM-2 inhibitor AMG 232 (NRG DT001 trial, NCT03217266) or trabectedin (TRASTS trial, NCT02275286), are given as a supplement to neoadjuvant RT [56,57]. Imaging-defined high-risk patients could benefit from such additional therapies, 
whereas low-risk patients could be spared unnecessary toxicities. The true value of such radiomic-guided therapies should be investigated in future prospective trials.

Apart from direct prognostic assessment, radiomics may be beneficial for several other tasks in STS patients. For instance, tumor characterization in terms of molecular aberrations ("radiogenomics") or histological properties could be a potential outcome target. Multiple authors demonstrated noninvasive prediction of the important prognostic factor, "tumor grading" [58,59]. In an ongoing work, we could demonstrate promising results differentiating benign lipomas from atypical lipomatous tumors based on the murine double minutes (MDM2) gene amplification status. Besides, tumor response prediction may be another area of investigation by analyzing longitudinal changes in radiomic features ("delta radiomics") parallel to RT or systemic therapies [60]. The first studies in STS and osteosarcomas demonstrated promising results [61,62].

Multiple limitations of the study should be noted that leave room for improvement of radiomic models. First, both study cohorts were collected retrospectively, constituting a reason for a potential source of bias [63]. Second, to achieve clinically homogenous patient cohorts, the patient number had to be reduced and this impaired statistical power. Third, as in many multicenter radiomic studies, the patient cohorts are prone to a substantial amount of technical heterogeneity, including a large plethora of MRI scanner types and imaging protocols. Fourth, the heterogenous histologies of STS may impair better prognostic performance for semantic, but also radiomic models. Finally, the semantic imaging features in the training and testing cohort were read by three separate readers. However, one reader read a part of each cohort and thus may have falsely increased the likeliness of overoptimistic validation between cohorts. By addressing these limitations, future research may be able to develop more effective prognostic models. As consequence, an optimal study would comprise of a large prospectively acquired patient cohort, be restricted to a predefined STS histology type, and use clearly defined MRI acquisition protocols.

\section{Conclusions}

In conclusion, we demonstrated that both MRI-based radiomic features and semantic imaging features were associated with overall survival. For radiomic models, we found that a T2FS-based radiomic model enabled prognostic assessment in addition to previous work using T1FSGd. Both models were able to achieve significant patient stratification. In comparison, the semantic model showed a decreased performance in the testing cohort. Combined semantic + radiomic models did not improve performance. Further investigation is warranted to advance towards a more personalized approach for risk-adapted tailored treatment intensification or deintensification based on imaging-based biomarkers.

Supplementary Materials: The following are available online at https:/ / www.mdpi.com/article/10 .3390/cancers13081929/s1, Figure S1: Patient workflow, Figure S2: Time-dependent AUC curves, Figure S3: Calibration curves, Table S1: Histologies of soft tissue sarcomas, Table S2: MRI acquisition parameters, Table S3: Extracted radiomic features, Table S4: R packages, Table S5: Inter-reader agreement of semantic imaging features, Table S6: Performance comparison of machine learning models, Table S7: Selected features and model coefficients, Table S8: Brier scores of developed models, Table S9: Performance in non-independent cohorts, Table S10: TRIPOD checklist

Author Contributions: Conceptualization, J.C.P., H.C.W., P.L., M.J.N., A.S.G., and S.E.C.; methodology, J.C.P. and D.S.H.; software, J.C.P.; validation, M.J.N., A.S.G., H.D., and S.E.C.; formal analysis, J.C.P., J.N., Y.L., J.R.M., C.E.v.S., R.A., S.C.F., and O.K.; investigation, J.C.P.; resources, M.J.N., N.A.M., A.S.G., and S.E.C.; data curation, J.C.P., J.N., M.B.S., S.K.S., and O.K.; writing-original draft preparation, J.C.P.; writing—review and editing, M.J.N., N.A.M., C.K., A.S.G., H.D., H.C.W., P.L., S.E.C., and S.K.S.; visualization, J.C.P.; supervision, H.C.W., P.L., M.J.N., and S.E.C.; project administration, J.C.P.; funding acquisition, J.C.P. and M.J.N. All authors have read and agreed to the published version of the manuscript.

Funding: This work was funded by physician-scientist programs of the medical faculty of the Technical University of Munich (KFF) and the Helmholtz Zentrum Muenchen. 
Institutional Review Board Statement: The study was conducted according to the guidelines of the Declaration of Helsinki, and approved by the Institutional Review Board (or Ethics Committee) of the Technical University of Munich (protocol code 466/16s and date of approval: 28th March 2019.

Informed Consent Statement: Informed consent was obtained from all subjects involved in the study.

Data Availability Statement: The data presented in this study are available on request from the corresponding author dependent on ethics board approval. The data are not publicly available due to data protection legislation.

Acknowledgments: We sincerely thank Chapman T and Macomber M for segmentations of VOIs at UW.

Conflicts of Interest: The authors declare no conflict of interest.

\section{References}

1. Gutierrez, J.C.; Perez, E.A.; Franceschi, D.; Moffat, F.L.; Livingstone, A.S.; Koniaris, L.G. Outcomes for Soft-Tissue Sarcoma in 8249 Cases from a Large State Cancer Registry. J. Surg. Res. 2007, 141, 105-114. [CrossRef] [PubMed]

2. Gerrand, C.H.; Rankin, K. The treatment of soft-tissue sarcomas of the extremities. Prospective randomized evaluations of (1) limb-sparing surgery plus radiation therapy compared with amputation and (2) the role of adjuvant chemotherapy. Class. Pap. Orthop. 2014, 483-484. [CrossRef]

3. Koshy, M.; Rich, S.; Mohiuddin, M. Improved Survival with Radiation Therapy in High Grade Soft Tissue Sarcomas of the Extremities: A SEER Analysis. Int. J. Radiat. Oncol. Biol. Phys. 2013, 77, 1-15. [CrossRef] [PubMed]

4. Alektiar, K.M.; Brennan, M.F.; Healey, J.H.; Singer, S. Impact of intensity-modulated radiation therapy on local control in primary soft-tissue sarcoma of the extremity. J. Clin. Oncol. 2008, 26, 3440-3444. [CrossRef]

5. $\quad$ Peeken, J.C.; Knie, C.; Kessel, K.A.; Habermehl, D.; Kampfer, S.; Dapper, H.; Devecka, M.; Von Eisenhart-rothe, R.; Specht, K.; Weichert, W.; et al. Neoadjuvant image-guided helical intensity modulated radiotherapy of extremity sarcomas-A single center experience. Radiat. Oncol. 2019, 14, 4-11. [CrossRef]

6. Muehlhofer, H.M.L.; Schlossmacher, B.; Lenze, U.; Lenze, F.; Burgkart, R.; Gersing, A.S.; Peeken, J.C.; Combs, S.E.; Von EisenhartRothe, R.; Knebel, C. Oncological Outcome and Prognostic Factors of Surgery for Soft Tissue Sarcoma After Neoadjuvant or Adjuvant Radiation Therapy: A Retrospective Analysis over 15 Years. Anticancer Res. 2021, 41, 359-368. [CrossRef]

7. Peeken, J.C.; Goldberg, T.; Knie, C.; Komboz, B.; Bernhofer, M.; Pasa, F.; Kessel, K.A.; Tafti, P.D.; Rost, B.; Nüsslin, F.; et al. Treatment-related features improve machine learning prediction of prognosis in soft tissue sarcoma patients. Strahlentherapie Onkol. 2018, 194, 824-834. [CrossRef]

8. Peeken, J.C.; Hesse, J.; Haller, B.; Kessel, K.A.; Nüsslin, F.; Combs, S.E. Semantic imaging features predict disease progression and survival in glioblastoma multiforme patients. Strahlenther. Onkol. 2018, 194, 580-590. [CrossRef]

9. Wu, G.; Woodruff, H.C.; Sanduleanu, S.; Refaee, T.; Jochems, A.; Leijenaar, R.; Gietema, H.; Shen, J.; Wang, R.; Xiong, J.; et al. Preoperative CT-based radiomics combined with intraoperative frozen section is predictive of invasive adenocarcinoma in pulmonary nodules: A multicenter study. Eur. Radiol. 2020, 30, 2680-2691. [CrossRef]

10. Peeken, J.C.; Goldberg, T.; Pyka, T.; Bernhofer, M.; Wiestler, B.; Kessel, K.A.; Tafti, P.D.; Nüsslin, F.; Braun, A.E.; Zimmer, C.; et al. Combining multimodal imaging and treatment features improves machine learning-based prognostic assessment in patients with glioblastoma multiforme. Cancer Med. 2019, 8, 128-136. [CrossRef]

11. Crombé, A.; Marcellin, P.J.; Buy, X.; Stoeckle, E.; Brouste, V.; Italiano, A.; Le Loarer, F.; Kind, M. Soft-tissue sarcomas: Assessment of MRI features correlating with histologic grade and patient outcome. Radiology 2019, 291, 710-721. [CrossRef]

12. Peeken, J.C.; Nüsslin, F.; Combs, S.E. "Radio-oncomics"-The potential of radiomics in radiation oncology. Strahlenther. Onkol. 2017, 193, 767-779. [CrossRef]

13. Peeken, J.C.; Wiestler, B.; Combs, S.E. The potential of radiomics in clinical application. In Image Guided Radiooncology; Debus, J., Schober, O., Kiessling, F., Eds.; Springer: Berlin/Heidelberg, Germany, 2020.

14. Peeken, J.C.; Bernhofer, M.; Wiestler, B.; Goldberg, T.; Cremers, D.; Rost, B.; Wilkens, J.J.; Combs, S.E.; Nüsslin, F. Radiomics in radiooncology-Challenging the medical physicist. Phys. Med. 2018, 48, 27-36. [CrossRef]

15. Lambin, P.; Rios-Velazquez, E.; Leijenaar, R.; Carvalho, S.; van Stiphout, R.G.P.M.; Granton, P.; Zegers, C.M.L.; Gillies, R.; Boellard, R.; Dekker, A.; et al. Radiomics: Extracting more information from medical images using advanced feature analysis. Eur. J. Cancer 2012, 48, 441-446. [CrossRef]

16. Aerts, H.J.W.L.; Velazquez, E.R.; Leijenaar, R.T.H.; Parmar, C.; Grossmann, P.; Carvalho, S.; Cavalho, S.; Bussink, J.; Monshouwer, R.; Haibe-Kains, B.; et al. Decoding tumour phenotype by noninvasive imaging using a quantitative radiomics approach. Nat. Commun. 2014, 5, 4006. [CrossRef]

17. Rios Velazquez, E.; Parmar, C.; Liu, Y.; Coroller, T.P.; Cruz, G.; Stringfield, O.; Ye, Z.; Makrigiorgos, M.; Fennessy, F.; Mak, R.H.; et al. Somatic mutations drive distinct imaging phenotypes in lung cancer. Cancer Res. 2017, 77, 3922-3930. [CrossRef]

18. Diehn, M.; Nardini, C.; Wang, D.S.; McGovern, S.; Jayaraman, M.; Liang, Y.; Aldape, K.; Cha, S.; Kuo, M.D. Identification of noninvasive imaging surrogates for brain tumor gene-expression modules. Proc. Natl. Acad. Sci. USA 2008, 105, 5213-5218. [CrossRef] 
19. Peeken, J.C.; Shouman, M.A.; Kroenke, M.; Rauscher, I.; Maurer, T.; Gschwend, J.E.; Eiber, M.; Combs, S.E. A CT-based radiomics model to detect prostate cancer lymph node metastases in PSMA radioguided surgery patients. Eur. J. Nucl. Med. Mol. Imaging 2020, 47, 2968-2977. [CrossRef]

20. Peeken, J.C.; Molina-Romero, M.; Diehl, C.; Menze, B.H.; Straube, C.; Meyer, B.; Zimmer, C.; Wiestler, B.; Combs, S.E. Deep learning derived tumor infiltration maps for personalized target definition in Glioblastoma radiotherapy. Radiother. Oncol. 2019, 138, 166-172. [CrossRef]

21. Leger, S.; Zwanenburg, A.; Leger, K.; Lohaus, F.; Linge, A.; Schreiber, A.; Kalinauskaite, G.; Tinhofer, I.; Guberina, N.; Guberina, M.; et al. Comprehensive Analysis of Tumour Sub-Volumes for Radiomic Risk Modelling in Locally Advanced HNSCC. Cancers 2020, 12, 3047. [CrossRef]

22. Spraker, M.B.; Wootton, L.S.; Hippe, D.S.; Ball, K.C.; Peeken, J.C.; Macomber, M.W.; Chapman, T.R.; Hoff, M.; Kim, E.Y.; Pollack, S.M.; et al. MRI Radiomic Features Are Independently Associated with Overall Survival in Soft Tissue Sarcoma. Adv. Radiat. Oncol. 2019, 4, 413-421. [CrossRef]

23. Vallières, M.; Freeman, C.R.; Skamene, S.R.; El Naqa, I. A radiomics model from joint FDG-PET and MRI texture features for the prediction of lung metastases in soft-tissue sarcomas of the extremities. Phys. Med. Biol. 2015, 60, 5471-5496. [CrossRef]

24. Moons, K.G.M.; Altman, D.G.; Reitsma, J.B.; Ioannidis, J.P.A.; Macaskill, P.; Steyerberg, E.W.; Vickers, A.J.; Ransohoff, D.F.; Collins, G.S. Transparent Reporting of a multivariable prediction model for Individual Prognosis or Diagnosis (TRIPOD): Explanation and Elaboration. Ann. Intern. Med. 2015, 162, W1-W73. [CrossRef]

25. Fedorov, A.; Beichel, R.; Kalphaty-Cramer, J.; Finet, J.; Fillion-Robbin, J.-C.; Pujol, S.; Bauer, C.; Jennings, D.; Fennessy, F.; Sonka, M.; et al. 3D slicers as an image computing platform for the quantitative imaging network. Magn. Reson. Imaging 2012, 30, 1323-1341. [CrossRef]

26. Tustison, N.J.; Gee, J.C. N4ITK: Nick's N3 ITK Implementation for MRI Bias Field Correction. Insight J. 2009, 9, 1-8.

27. Van Griethuysen, J.J.M.; Fedorov, A.; Parmar, C.; Hosny, A.; Aucoin, N.; Narayan, V.; Beets-Tan, R.G.H.; Fillion-Robin, J.-C.; Pieper, S.; Aerts, H.J.W.L. Computational Radiomics System to Decode the Radiographic Phenotype. Cancer Res. 2017, 77, e104-e107. [CrossRef]

28. Zwanenburg, A.; Vallières, M.; Abdalah, M.A.; Aerts, H.J.W.L.; Andrearczyk, V.; Apte, A.; Ashrafinia, S.; Bakas, S.; Beukinga, R.J.; Boellaard, R.; et al. The Image Biomarker Standardization Initiative: Standardized Quantitative Radiomics for High-Throughput Image-based Phenotyping. Radiology 2020, 191145. [CrossRef]

29. Depeursinge, A.; Andrearczyk, V.; Whybra, P.; van Griethuysen, J.; Müller, H.; Schaer, R.; Vallières, M.; Zwanenburg, A. Standardised convolutional filtering for radiomics. arXiv 2020, arXiv:2006.05470.

30. Steiger, P.; Sood, R. How Can Radiomics Be Consistently Applied across Imagers and Institutions? Radiology 2019, $291,60-61$. [CrossRef]

31. Johnson, W.E.; Li, C.; Rabinovic, A. Adjusting batch effects in microarray expression data using empirical Bayes methods. Biostatistics 2007, 8, 118-127. [CrossRef]

32. Lucia, F.; Visvikis, D.; Vallières, M.; Desseroit, M.; Miranda, O.; Robin, P.; Bonaffini, P.A.; Alfieri, J.; Masson, I.; Mervoyer, A.; et al. External validation of a combined PET and MRI radiomics model for prediction of recurrence in cervical cancer patients treated with chemoradiotherapy. Eur. J. Nucl. Med. Mol. Imaging 2019, 46, 864-877. [CrossRef] [PubMed]

33. Orlhac, F.; Frouin, F.; Nioche, C.; Ayache, N.; Buvat, I. Validation of A Method to Compensate Multicenter Effects Affecting CT Radiomics. Radiology 2019, 291, 53-59. [CrossRef] [PubMed]

34. Fortin, J.; Parker, D.; Tunç, B.; Watanabe, T.; Elliott, M.A.; Ruparel, K.; Roalf, D.R.; Satterthwaite, T.D.; Gur, R.C.; Gur, R.E.; et al. NeuroImage Harmonization of multi-site diffusion tensor imaging data. Neuroimage 2017, 161, 149-170. [CrossRef] [PubMed]

35. Holzapfel, K.; Regler, J.; Baum, T.; Rechl, H.; Specht, K.; Haller, B.; von Eisenhart-Rothe, R.; Gradinger, R.; Rummeny, E.J.; Woertler, K. Local Staging of Soft-Tissue Sarcoma: Emphasis on Assessment of Neurovascular Encasement-Value of MR Imaging in 174 Confirmed Cases. Radiology 2015, 275, 501-509. [CrossRef]

36. Petscavage-Thomas, J.M.; Walker, E.A.; Logie, C.I.; Clarke, L.E.; Duryea, D.M.; Murphey, M.D. Soft-tissue myxomatous lesions: Review of salient imaging features with pathologic comparison. Radiographics 2014, 34, 964-980. [CrossRef]

37. Yoo, H.J.; Hong, S.H.; Kang, Y.; Choi, J.-Y.; Moon, K.C.; Kim, H.-S.; Han, I.; Yi, M.; Kang, H.S. MR imaging of myxofibrosarcoma and undifferentiated sarcoma with emphasis on tail sign; diagnostic and prognostic value. Eur. Radiol. 2014, 24, 1749-1757. [CrossRef]

38. Knebel, C.; Neumann, J.; Schwaiger, B.J.; Karampinos, D.C.; Pfeiffer, D.; Specht, K.; Lenze, U.; Von Eisenhart-Rothe, R.; Rummeny, E.J.; Woertler, K.; et al. Differentiating atypical lipomatous tumors from lipomas with magnetic resonance imaging: A comparison with MDM2 gene amplification status. BMC Cancer 2019, 19, 1-8. [CrossRef]

39. Tibshirani, R. Regression Shrinkage and Selection Via the Lasso. J. R. Stat. Soc. Ser. B 1996, 58, 267-288. [CrossRef]

40. Ishwaran, H.; Gerds, T.A.; Kogalur, U.B.; Moore, R.D.; Gange, S.J.; Lau, B.M. Random survival forests for competing risks. Biostatistics 2014, 15, 757-773. [CrossRef]

41. Waldron, L.; Pintilie, M.; Tsao, M.S.; Shepherd, F.A.; Huttenhower, C.; Jurisica, I. Optimized application of penalized regression methods to diverse genomic data. Bioinformatics 2011, 27, 3399-3406. [CrossRef]

42. AJCC. Cancer Staging Manual, 8th ed.; Amin, M.B., Edge, S., Greene, F., Byrd, D.R., Brookland, R.K., Washington, M.K., Gershenwald, J.E., Compton, C.C., Hess, K.R., Sullivan, D.C., et al., Eds.; Springer International Publishing: New York, NY, USA, 2017. 
43. Kursa, M.B.; Rudnicki, W.R. Feature selection with the boruta package. J. Stat. Softw. 2010, 36, 1-13. [CrossRef]

44. Wu, G.; Woodruff, H.C.; Shen, J.; Refaee, T.; Sanduleanu, S.; Abdalla, I.; Leijenaar, R.T.H.; Wang, R.; Xiong, J.; Bian, J.; et al. Diagnosis of Invasive Lung Adenocarcinoma Based on Chest CT Radiomic Features of Part-Solid Pulmonary Nodules: A Multicenter Study. Radiology 2020, 192431. [CrossRef]

45. Lang, M.; Binder, M.; Richter, J.; Schratz, P.; Pfisterer, F.; Coors, S.; Au, Q.; Casalicchio, G.; Kotthoff, L.; Bischl, B. mlr3: A modern object-oriented machine learning framework in R. J. Open Source Softw. 2019, 4, 1903. [CrossRef]

46. Landis, J.R.; Koch, G.G. The Measurement of Observer Agreement for Categorical Data. Biometrics 1977, 33, 159. [CrossRef]

47. Altman, D.G. Practical Statistics for Medical Research; Chapman and Hall/CRC: Boca Raton, FL, USA, 2018; ISBN 978-0-41227-630-9.

48. Wortman, J.R.; Tirumani, S.H.; Jagannathan, J.P.; Tirumani, H.; Shinagare, A.B.; Hornick, J.L.; Ramaiya, N.H. Primary Extremity Liposarcoma: MRI Features, Histopathology, and Clinical Outcomes. J. Comput. Assist. Tomogr. 2016, 40, 791-798. [CrossRef]

49. Mühlhofer, H.; Gersing, A.; Pfeiffer, D.; Wörtler, K.; Lenze, U.; Lenze, F.; Lallinger, V.; Haller, B.; Burgkart, R.; von Eisenhart-Rothe, R.; et al. Preoperative evaluation of myxofibrosarcoma: Prognostic value and reproducibility of different features on MRI. Anticancer Res. 2020, 40, 5793-5800. [CrossRef]

50. Zwanenburg, A.; Löck, S. Why validation of prognostic models matters? Radiother. Oncol. 2018, 127, 370-373. [CrossRef]

51. Peeken, J.C.; Bernhofer, M.; Spraker, M.B.; Pfeiffer, D.; Devecka, M.; Thamer, A.; Shouman, M.A.; Ott, A.; Nüsslin, F.; Mayr, N.A.; et al. CT-based radiomic features predict tumor grading and have prognostic value in patients with soft tissue sarcomas treated with neoadjuvant radiation therapy. Radiother. Oncol. 2019, 135, 187-196. [CrossRef]

52. Zer, A.; Prince, R.M.; Amir, E.; Abdul Razak, A.R. Multi-agent chemotherapy in advanced soft tissue sarcoma (STS)—A systematic review and meta-analysis. Cancer Treat. Rev. 2018, 63, 71-78. [CrossRef]

53. Wong, P.; Houghton, P.; Kirsch, D.G.; Finkelstein, S.E.; Monjazeb, A.M.; Xu-Welliver, M.; Dicker, A.P.; Ahmed, M.; Vikram, B.; Teicher, B.A.; et al. Combining targeted agents with modern radiotherapy in soft tissue sarcomas. J. Natl. Cancer Inst. 2014, 106, 16-18. [CrossRef]

54. Schwartz, G.K.; Tap, W.D.; Qin, L.-X.; Livingston, M.B.; Undevia, S.D.; Chmielowski, B.; Agulnik, M.; Schuetze, S.M.; Reed, D.R.; Okuno, S.H.; et al. Cixutumumab and temsirolimus for patients with bone and soft-tissue sarcoma: A multicentre, open-label, phase 2 trial. Lancet Oncol. 2013, 14, 371-382. [CrossRef]

55. Pollack, S.M.; He, Q.; Yearley, J.H.; Emerson, R.; Vignali, M.; Zhang, Y.; Redman, M.W.; Baker, K.K.; Cooper, S.; Donahue, B.; et al. T-cell infiltration and clonality correlate with programmed cell death protein 1 and programmed death-ligand 1 expression in patients with soft tissue sarcomas. Cancer 2017, 123, 3291-3304. [CrossRef]

56. Gronchi, A.; Hindi, N.; Cruz, J.; Blay, J.-Y.; Sanfilippo, R.; Morosi, C.; Romero, J.; Peinado, J.; Lopez-Pousa, A.; Alvarez Alvarez, R.M.; et al. Trabectedin and radiotherapy in soft-tissue sarcoma (TRASTS) study: An international, prospective, phase I/II trial-A collaborative Spanish (GEIS), Italian (ISG), and French (FSG) groups study. J. Clin. Oncol. 2017, 35, 11061. [CrossRef]

57. Gluck, W.L.; Gounder, M.M.; Frank, R.; Eskens, F.; Blay, J.Y.; Cassier, P.A.; Soria, J.-C.; Chawla, S.; de Weger, V.; Wagner, A.J.; et al. Phase 1 study of the MDM2 inhibitor AMG 232 in patients with advanced P53 wild-type solid tumors or multiple myeloma. Investig. New Drugs 2020, 38, 831-843. [CrossRef]

58. Peeken, J.C.; Spraker, M.B.; Knebel, C.; Dapper, H.; Pfeiffer, D.; Devecka, M.; Thamer, A.; Shouman, M.A.; Ott, A.; von Eisenhart-Rothe, R.; et al. Tumor grading of soft tissue sarcomas using MRI-based radiomics. EBioMedicine 2019, 48, 332-340. [CrossRef]

59. Yan, R.; Hao, D.; Li, J.; Liu, J.; Hou, F.; Chen, H.; Duan, L.; Huang, C.; Wang, H.; Yu, T. Magnetic Resonance Imaging-Based Radiomics Nomogram for Prediction of the Histopathological Grade of Soft Tissue Sarcomas: A Two-Center Study. J. Magn. Reson. Imaging 2021. [CrossRef]

60. Gennaro, N.; Reijers, S.; Bruining, A.; Messiou, C.; Haas, R.; Colombo, P.; Bodalal, Z.; Beets-Tan, R.; van Houdt, W.; van der Graaf, W.T.A. Imaging response evaluation after neoadjuvant treatment in soft tissue sarcomas: Where do we stand? Crit. Rev. Oncol. Hematol. 2021, 160, 103309. [CrossRef]

61. Crombé, A.; Périer, C.; Kind, M.; De Senneville, B.D.; Le Loarer, F.; Italiano, A.; Buy, X.; Saut, O. T2-based MRI Delta-radiomics improve response prediction in soft-tissue sarcomas treated by neoadjuvant chemotherapy. J. Magn. Reson. Imaging 2018, 1-14. [CrossRef]

62. Lin, P.; Yang, P.F.; Chen, S.; Shao, Y.Y.; Xu, L.; Wu, Y.; Teng, W.; Zhou, X.Z.; Li, B.H.; Luo, C.; et al. A Delta-radiomics model for preoperative evaluation of Neoadjuvant chemotherapy response in high-grade osteosarcoma. Cancer Imaging 2020, $20,7$. [CrossRef] [PubMed]

63. Sica, G.T. Bias in Research Studies. Radiology 2006, 238, 780-789. [CrossRef] [PubMed] 